\title{
Spectroscopic fingerprints of work-function-controlled phthalocyanine charging on metal surfaces
}

\author{
P. Borghetti, ${ }^{*}, 1,2$ A. El-Sayed, ${ }^{2,3}$ E. Goiri, ${ }^{1,2}$ C. Rogero, ${ }^{1,2} J$. Lobo-Checa, ${ }^{2}$ L. Floreano, ${ }^{4}$ J. E. \\ Ortega, ${ }^{*}, 1,2,3$. D. G. de Oteyza ${ }^{*, 1,2}$ \\ ${ }^{1}$ Donostia International Physics Center, Paseo Manuel Lardizabal 4, San Sebastián, Spain \\ ${ }^{2}$ Centro de Fisica de Materiales (CSIC-UPV/EHU)-Materials Physics Center (MPC), San \\ Sebastián, Spain \\ ${ }^{3}$ Universidad del Pais Vasco, Dpto. Física Aplicada I, San Sebastián, Spain \\ ${ }^{4}$ CNR-IOM, Laboratorio Nazionale TASC, Basovizza SS-14 Km. 163.5, Trieste, Italy \\ * e-mail: patrizia_borghetti@ehu.es; enrique.ortega@ehu.es; d_g_oteyza@ehu.es
}

\begin{abstract}
.
The electronic character of a $\pi$-conjugated molecular overlayer on a metal surface can change from semiconducting to metallic, depending on how molecular orbitals arrange with respect to the electrode's Fermi level. Molecular level alignment is thus a key property that strongly influences the performance of organic-based devices. In this work we report how the electronic level alignment of copper-phthalocyanines on metal surfaces can be tailored by controlling the substrate work function. We even show the way of finely tuning it for one fixed phthalocyaninemetal combination, such that charge transfer into empty molecular levels can be triggered across the metal-organic interface. These intriguing observations are the outcome of a powerful combination of surface-sensitive electron spectroscopies, which reveal a number of characteristic spectroscopic fingerprints that provide a fully coherent picture of the physical chemistry phenomena occurring at these relevant interfaces.
\end{abstract}


Energy-level alignment at hetero-interfaces plays an essential role in the functionality of charge injection devices, such as organic solar cells, where the efficiency is largely determined by the relative band edge positions of all its constituents. By way of example, band alignment at the metal/semiconductor interface critically affects the contact resistance, which often limits device performance. ${ }^{1}$ In fact, an appropriate level alignment may drive the formation of hybrid interface states at the Fermi energy, trigger significant charge transfer and lower interface potential barriers. ${ }^{2,3}$ Electrical doping ${ }^{4}$ and/or interface engineering ${ }^{1,5-8}$ are thus essential to optimize the relative energies of the electronic states involved in the conduction within each of the materials: (i) the states around the Fermi level in the metal and (ii) the valence or conduction bands in the semiconductor. ${ }^{9,10}$ For the particular case of organic semiconductors, upon which increasingly high hopes are being placed as future active components of a variety of devices, the critical states correspond to the highest occupied (HOMO) and lowest unoccupied molecular orbitals (LUMO). Their alignment with respect to the substrate's Fermi level has therefore been extensively studied over the last decades for many molecule-metal combinations. Initially it was assumed that the energetics was governed by vacuum level alignment according to the SchottkyMott model. Within this model, for a given molecule with specific ionization potential and electron affinity, the charge injection barriers are simply defined by the electrode's work function. It was soon found, however, that much more complex processes are involved, including the generation of interface dipoles, charge transfer or interface hybridizations. ${ }^{2,3,11,12}$ For all that, the electrode's work function remains, although in a less predictable manner, a key parameter with which to influence the energy level alignment. Most importantly, the work function can be easily modified experimentally, typically by changing the substrate material, the surface orientation or by additional surface functionalization. 
In this work, we apply this concept to phthalocyanine-based metal-organic interfaces. Today copper-phthalocyanine $\mathrm{CuPc}$, an archetypal heteroaromatic dye, as well as its fluorinated counterpart $\mathrm{F}_{16} \mathrm{CuPc},{ }^{13}$ are widely studied due to their electronic properties, processing flexibility, and to the promising efficiencies of phthalocyanine-based optoelectronic devices. Using a thorough electron spectroscopy approach, we characterize the electronic levels of copper-phthalocyanines at the interface with coinage metals. As a result of the decreasing work function when changing from $\mathrm{Au}(111)$, through $\mathrm{Cu}(111)$ to $\mathrm{Ag}(111)$, we observe a tunable energy level alignment, including a controllable charge transfer to the molecular LUMO. Our spectroscopic analysis reveals that transferring charge from the substrate lifts the LUMO degeneracy, breaking the four-fold symmetry of the phthalocyanine. Most remarkably, we show that this charge transfer into the LUMO can be tuned even at the very same metal-organic interface by making use of the supramolecular environment-dependent work function in molecular blends. ${ }^{14,15}$

\section{Results}

The different interface energy level alignment of $\mathrm{F}_{16} \mathrm{CuPc}$ monolayers on various coinage metal surfaces is summarized schematically in Fig. 1a . From photoemission experiments described in more detail below, we extract the HOMO level positions, as well as the work functions of $\mathrm{Au}(111), \mathrm{Cu}(111)$ and $\mathrm{Ag}(111)$ surfaces before and after being covered by full $\mathrm{F}_{16} \mathrm{CuPc}$ monolayers (ML). The latter include the interface dipoles and turn out to be the truly relevant magnitudes, amounting to $5.15 \pm 0.03 \mathrm{eV}, 4.67 \pm 0.03 \mathrm{eV}$ and $4.40 \pm 0.03 \mathrm{eV}$ on the three respective substrates. In this probed range, the Fermi level $\left(E_{F}\right)$ excursion within the molecular semiconducting gap neither reaches the HOMO level nor its high-energy tail. The LUMO energies were not accessed experimentally but can be roughly estimated by summing the 
HOMO-LUMO gap energy to that of the HOMO. Taking a typically reported gap value for $\mathrm{F}_{16} \mathrm{CuPc}$ of $\sim 1.7 \mathrm{eV}$ implies that $E_{F}$ is centered in the gap for the $\mathrm{Au}(111)$ case, approaching the LUMO as the work function is reduced. In fact, photoemission data show that on $\operatorname{Ag}(111) E_{F}$ crosses the low energy tail of the LUMO level, inducing a partial charge transfer from substrate to molecule. As shown in Fig. 1a and in analogy to the previously reported findings upon charging of the closely related CuPc, ${ }^{16}$ the two-fold degeneracy of the $\mathrm{F}_{16} \mathrm{CuPc} e_{g}$ LUMO levels (displayed in Fig. 1b) is thus lifted, likely by a Jahn-Teller effect.

The lifted degeneracy of LUMO levels is best observed by probing the unoccupied molecular orbitals by means of NEXAFS. Spectra of the $\mathrm{C}$ K-edge of $\mathrm{F}_{16} \mathrm{CuPc}$ monolayers on the three different surfaces are displayed in Fig. 1c. On $\mathrm{Au}(111)$ and $\mathrm{Cu}(111)$ the spectra closely resemble that of a multilayer, ${ }^{13}$ indicating that contact with these surfaces doesn't significantly alter the unoccupied states of $\mathrm{F}_{16} \mathrm{CuPc}$. By contrast, on $\mathrm{Ag}(111)$ significant modifications are found in the low energy part of the spectrum: two additional components $\left(\mathrm{C}_{\mathrm{A}^{\prime}}\right.$, and $\mathrm{C}_{\mathrm{B}}$ ) appear at the low photon energy side of peaks $\mathrm{C}_{\mathrm{A}}$ and $\mathrm{C}_{\mathrm{B}}$. According to previous findings on $\mathrm{F}_{16} \mathrm{CuPc}$ thick films, ${ }^{17} \mathrm{C}_{\mathrm{A}}$ and $\mathrm{C}_{\mathrm{B}}$ correspond to transitions from the $\mathrm{C}$ 1s core levels on the $\mathrm{C}-\mathrm{C}$ phenyl and $\mathrm{C}$ $\mathrm{N}$ pyrrole sites, respectively, to the LUMO. A plausible explanation to the spectral changes is the association of the new additional components at lower photon energy $\left(\mathrm{C}_{\mathrm{A}^{\prime}}\right.$, and $\left.\mathrm{C}_{\mathrm{B}^{\prime}}\right)$ and the main absorption lines $\left(\mathrm{C}_{\mathrm{A}}\right.$ and $\left.\mathrm{C}_{\mathrm{B}}\right)$ with transitions into the low and high energy components of the split $e_{g}$ LUMO levels, respectively. The partial occupation of the lower energy LUMO level also agrees with the lower intensity of the new components, while the absence of similar fingerprints on subsequent resonances is explained by the convolution with absorption transitions onto higher energy unoccupied orbitals unaffected by the Jahn-Teller distortion. ${ }^{17}$ 
Comparison of the valence band spectra of $\mathrm{F}_{16} \mathrm{CuPc}$ on $\mathrm{Au}(111)$ and $\mathrm{Ag}(111)$, displayed in Fig. 2a, supports the above described scenario. That is, lowering the work function when going from $\mathrm{Au}(111)$ to $\mathrm{Ag}(111)$ does not only increase the HOMO binding energy from 0.85 to 1.30 $\mathrm{eV}$, but also causes a partial filling of the LUMO level (labeled as F-LUMO) on $\operatorname{Ag}(111)$. This is observable as an increase in the photoemission intensity close to the Fermi level with respect to the signal on the clean substrate, whereas it is absent for $\mathrm{F}_{16} \mathrm{CuPc}$ on $\mathrm{Au}(111)$. Fitting of the spectrum on $\mathrm{Ag}(111)$ (included in Fig. 2a) renders a F-LUMO centered at $0.15 \mathrm{eV}$ and clearly truncated at the Fermi edge, indicative of the partial filling that leaves another part of the level empty and accessible to NEXAFS experiments.

The associated N K-edge NEXAFS spectra taken under p- and s-polarization on $\mathrm{F}_{16} \mathrm{CuPc}$ monolayers on $\mathrm{Au}(111)$ and $\mathrm{Ag}(111)$ are displayed in Fig. 2b. The most remarkable difference among both substrates is the appearance on $\mathrm{Ag}(111)$ of a clear shoulder $\mathrm{N}_{\mathrm{A}^{\prime}}$, at $\mathrm{hv}=397.5 \mathrm{eV}$, just at the low energy side of the first main peak $\mathrm{N}_{\mathrm{A}}$ associated with transitions into the LUMO. This feature $\mathrm{N}_{\mathrm{A}}$, is neither observed in the spectra on $\mathrm{Au}(111)$ nor in the multilayer and is ascribed, as in the C K-edge spectra (Fig. 1c), to the split and partially filled e $\mathrm{LUMO}$ level. As a result of the partial charge transfer, the total intensity obtained by summing the contributions of $\mathrm{N}_{\mathrm{A}}$ and $\mathrm{N}_{\mathrm{A}^{\prime}}$ on $\mathrm{Ag}(111)$ is still $15 \%$ less than the intensity of $\mathrm{N}_{\mathrm{A}}$ on $\mathrm{Au}(111)$. Considerable spectral modifications on $\operatorname{Ag}(111)$ are also detected in the photon energy range between 399 and $403 \mathrm{eV}$, where a shift of about $0.3 \mathrm{eV}$ to lower photon energy for the band $\mathrm{N}_{\mathrm{B}}$ and a broadening of band $\mathrm{N}_{\mathrm{C}}$ are observed (ascribed in analogy to CuPc to transitions into the LUMO+1 and LUMO+2, respectively $)^{18}$. The changes are analogous to those observed on $\mathrm{CuPc}$ upon $\mathrm{K}^{18}$ and $\mathrm{Rb}^{19}$ doping and thus provide further support to our proposed partial LUMO filling scenario. Spectral changes appear solely under p-polarization, underlining their origin in $\pi^{*}$ orbitals. Moreover, the 
complete quenching of the $\pi^{*}$ transitions in s-polarization, which has been measured also for the $\mathrm{C}$ K-edge, indicates a flat absorption configuration for the $\mathrm{F}_{16} \mathrm{CuPc}$ plane on both $\mathrm{Au}(111)$ and $\operatorname{Ag}(111)$ surfaces, as observed also by scanning tunneling microscopy. ${ }^{14,20,21}$ The equally flat orientation adopted by molecules on both surfaces thus guarantees that the differences observed between the NEXAFS spectra of the two systems stem from changes in the electronic structure rather than from a modified molecular orientation.

On $\mathrm{Cu}(111), \mathrm{F}_{16} \mathrm{CuPc}$ NEXAFS measurements at the $\mathrm{C}$ K-edge (Fig. 1c) and N K-edge (Fig. S1) show very similar spectral line shapes to those on $\mathrm{Au}(111)$, suggestive of an absence of charge transfer from substrate to molecule. Indeed, no intensity close to $\mathrm{E}_{\mathrm{F}}$ is found in valence band photoemission spectra of $\mathrm{F}_{16} \mathrm{CuPc} / \mathrm{Cu}(111)$ (Fig. 3a). However, from the similar HOMO binding energy and the relatively close work functions of $\mathrm{F}_{16} \mathrm{CuPc} / \mathrm{Cu}$ and $\mathrm{F}_{16} \mathrm{CuPc} / \mathrm{Ag}$ we expect the $\mathrm{F}_{16} \mathrm{CuPc} / \mathrm{Cu} \mathrm{LUMO}$ to be very close to $\mathrm{E}_{\mathrm{F}}$ (Fig. 1a). As recently reported, one way to fine-tune the sample's work function and thereby the energy-level alignment at the molecule/metal interface is to mix molecular species whose associated interface dipoles on a given surface differ significantly. ${ }^{14,22}$ This is typically the case e.g. with molecules of complementary donor and acceptor character. ${ }^{14,23}$ In the case of $\mathrm{F}_{16} \mathrm{CuPc}$, the molecular levels can be rigidly shifted to higher binding energies by mixing them for example with pentacene (PEN) molecules. On $\mathrm{Cu}(111), \mathrm{F}_{16} \mathrm{CuPc}: \mathrm{PEN}$ monolayer blends with a 1:2 stoichiometry form a particularly stable and easy to prepare crystalline structure, as determined by scanning tunneling microscopy (Fig. 3c). ${ }^{14}$ The work function of the $1: 2$ blend on $\mathrm{Cu}(111)$ is slightly lower than that of $\mathrm{F}_{16} \mathrm{CuPc} / \mathrm{Ag}(111)(\phi=4.28 \pm 0.03 \mathrm{eV}$ vs. $\phi=4.40 \pm 0.03 \mathrm{eV})$, and thus we expect comparable or even stronger interfacial charge transfer effects in the case of the 1:2 blend on $\mathrm{Cu}$. 
To confirm this assumption, valence band photoemission and NEXAFS spectra were measured for the blend. Fig. 3a shows valence band spectra of the 1:2 blend grown by sequential deposition of $0.5 \mathrm{ML}$ of PEN followed by $0.5 \mathrm{ML}$ of $\mathrm{F}_{16} \mathrm{CuPc}$ (note that due to the different molecular sizes a 1:1 ratio in surface area coverage corresponds to a 1:2 stoichiometry), although equivalent results can be obtained by co-depositing the two molecular species. The valence band spectrum of $0.5 \mathrm{ML}$ of PEN displays two peaks at $0.65 \mathrm{eV}$ (A) and $1.35 \mathrm{eV}$ (B). By adding 0.5 $\mathrm{ML}$ of $\mathrm{F}_{16} \mathrm{CuPc}$, peak $\mathrm{B}$ increases in intensity due to the additional emission of the $\mathrm{F}_{16} \mathrm{CuPc}$ HOMO, while a noticeable intensity A' appears at the low binding energy side of PEN's peak A. To highlight this feature, we subtract the spectrum of 0.5 ML PEN from that of the 1:2 blend (black line in bottom part of Fig. 3a) and compare it to the pure $\mathrm{F}_{16} \mathrm{CuPc}$ monolayer spectrum after subtraction of the $\mathrm{Cu}(111)$ reference spectrum (red line in bottom part of Fig. 3a). From this analysis, it is evident that the new structure appearing at about $0.30 \mathrm{eV}$ is characteristic neither of the PEN nor of $\mathrm{F}_{16} \mathrm{CuPc}$ pure layers but arises from mixing the two species. In view of the results on $\mathrm{F}_{16} \mathrm{CuPc} / \mathrm{Ag}(111)$, we ascribe this new feature to the partial filling of the $\mathrm{F}_{16} \mathrm{CuPc} \mathrm{LUMO}$ (FLUMO). Probing the unoccupied molecular orbitals, NEXAFS N K-edge spectra on $\mathrm{Cu}(111)$ change substantially if we compare pure $\mathrm{F}_{16} \mathrm{CuPc}$ layers with $\mathrm{F}_{16} \mathrm{CuPc}$ :PEN blends (Fig. $3 \mathrm{~b}$ ). As occurred for $\mathrm{F}_{16} \mathrm{CuPc}$ on $\mathrm{Ag}(111)$, in the blends on $\mathrm{Cu}(111)$ an absorption intensity $\mathrm{N}_{\mathrm{A}^{\prime}}$ appears at the low energy side of the main peak $\mathrm{N}_{\mathrm{A}}$, which in turn appears significantly reduced, $\mathrm{N}_{\mathrm{B}}$ shifts down in energy and $\mathrm{N}_{\mathrm{C}}$ broadens. All the above is in line with a partial filling of the LUMO level and the consequent lifting of its degeneracy as displayed schematically in Fig. 3c.

It is worth mentioning that the new peak emerging in the valence band spectrum of the 1:2 blend on $\mathrm{Cu}(111)$ is detected at $0.15 \mathrm{eV}$ higher binding energy than the corresponding peak of $\mathrm{F}_{16} \mathrm{CuPc} / \mathrm{Ag}(111)$. This is in fair agreement with the difference in work function measured for 
the two systems, which underlines the fundamental role of vacuum level shifts in moving $\mathrm{F}_{16} \mathrm{CuPc}$ frontier orbitals below $\mathrm{E}_{\mathrm{F}}$ and further demonstrates the possibility of fine-tuning charge transfer across phthalocyanine-metal interfaces by work function manipulation. Indeed, in accordance with the smaller work function value and larger binding energy of the F-LUMO feature, a stronger degree of LUMO filling is deduced for the blend on $\mathrm{Cu}(111)$, which is further confirmed by a stronger decrease in intensity in the NEXAFS N K-edge spectra of peaks $\mathrm{N}_{\mathrm{A}}$ and $\mathrm{N}_{\mathrm{A}^{\prime}}$ for 1:2 blend on $\mathrm{Cu}(111)$ than for those on $\mathrm{F}_{16} \mathrm{CuPc} / \mathrm{Ag}(111)$ (see SI, Fig. S1).

Core levels are sensitive to the charge state of the atoms they originate from, and consequently to molecular charging. Besides, they represent the initial states for the electronic transitions measured in NEXAFS experiments and thus have direct impact on those spectra. To deepen our understanding of the above described findings, we have measured $\mathrm{N}$ 1s and $\mathrm{C}$ 1s core level spectra of each of the systems discussed above and summarize them, along with their corresponding fits, in Fig. 4. While $\mathrm{F}_{16} \mathrm{CuPc}$ comprises two non-equivalent $\mathrm{N}$ atom sites and four $\mathrm{C}$ atom sites, for monolayers on $\mathrm{Au}(111)$, as well as for multilayers, satisfactory fits can be obtained by using only one component for the $\mathrm{N} 1$ s spectrum and three components for the $\mathrm{C} 1 \mathrm{~s}$ spectrum (together with their associated satellites). The latter correspond to $\mathrm{C}$ atoms bonded solely to $\mathrm{C}(\mathrm{C}-\mathrm{C}), \mathrm{C}$ atoms in the pyrrole rings bonded to $\mathrm{N}(\mathrm{C}-\mathrm{N})$, and $\mathrm{C}$ atoms bonded to $\mathrm{F}$ (CF), in order of increasing binding energy as the atoms loose more electrons to species of increasing electronegativity.13'14, ${ }^{17}$ The fitted intensity ratio for peaks C-C: C-N: C-F agrees well with the expected 1:1:2 ratio for this molecule. For $\mathrm{F}_{16} \mathrm{CuPc}$ on $\mathrm{Cu}(111)$ the spectra can be fitted with a similar model (Fig. 4), albeit with broader components stemming from a stronger molecule-substrate hybridization. As occurred with the HOMO, core levels are shifted by $0.5 \mathrm{eV}$ to higher binding energy with respect to $\mathrm{F}_{16} \mathrm{CuPc}$ on $\mathrm{Au}(111)$, which is consistent with the 
difference in work function measured for the two interfaces (Fig. 1a). More important changes are observed for the $\mathrm{F}_{16} \mathrm{CuPc} \mathrm{N}$ 1s and $\mathrm{C}$ 1s core level spectra as its LUMO gets partially filled in $\mathrm{F}_{16} \mathrm{CuPc} / \mathrm{Ag}(111)$ and $\mathrm{F}_{16} \mathrm{CuPc}: \mathrm{PEN} / \mathrm{Cu}(111)$. In these cases, the fitting procedure requires additional components to provide satisfactory results, evidencing an unambiguous splitting into two components with similar area behind the overall strongly broadened spectra. This splitting effect being related to the partial charging of the $\mathrm{F}_{16} \mathrm{CuPc} \mathrm{LUMO}$, it is strongest for the system with larger charge transfer, $\mathrm{F}_{16} \mathrm{CuPc}: \mathrm{PEN} / \mathrm{Cu}(111)$. The splitting's physical origin will be addressed in the discussion.

Finally, on the closely related sister molecule $\mathrm{CuPc}$, the same phenomenology is observed. Both molecules share similar electronic attributes, except for the larger electron affinity and ionization potential brought about by the molecular fluorination in $\mathrm{F}_{16} \mathrm{CuPc}^{13,21,24}$ However, the typically smaller interface dipole generated by fluorinated molecules ${ }^{14,23}$ leaves $\mathrm{F}_{16} \mathrm{CuPc}$ and $\mathrm{CuPc}$ with a comparable interfacial energy level alignment. As a consequence, the same spectral changes are observed when comparing CuPc's valence band (Fig. 5a) and core-level photoemission (Fig. 5c), as well as NEXAFS spectra (Fig. 5b), on $\mathrm{Au}(111)$ and $\operatorname{Ag}(111)$. On $\mathrm{Ag}(111)$, unambiguous intensity appears close to $\mathrm{E}_{\mathrm{F}}$ in the valence band photoemission spectra and corroborates partial filling of the LUMO (Fig. 5a), core-level spectra evidence their splitting into two components (Fig. 5c), and in N K-edge NEXAFS spectra a clear shoulder $\mathrm{N}_{\mathrm{A}}$, is observed at the low energy side of the $\mathrm{N}_{\mathrm{A}}$ LUMO resonance, the band $\mathrm{N}_{\mathrm{B}}$ is shifted down in energy and band $\mathrm{N}_{\mathrm{C}}$ is broadened. Altogether, we conclude the same scenario as for $\mathrm{F}_{16} \mathrm{CuPc}$ : as the work function is reduced in $\mathrm{Ag}(111)$, partial filling of the CuPc LUMO levels takes place and lifts their degeneracy (Fig. 5d). Noteworthy for our following discussion, the F-LUMO state is observed already at the sub-monolayer coverage ( $0.25 \mathrm{ML}$ and $0.55 \mathrm{ML})$ and grows in intensity 
as the coverage increases up to the ML (see SI, Fig. S2). Once the ML is formed, a further deposition of $\mathrm{CuPc}$ induces this peak to decrease, until it completely disappears at the nominal coverage of about 4 ML. Concurrently, the N 1s and C 1s spectra in the sub-ML to ML coverage regime are satisfactorily fitted by doubling the number of components, while at higher coverages the fit components simply reflect the stochiometry of the molecule (see SI, Fig. S2).

\section{Discussion}

The combined photoemission and absorption analysis of organic/metal interfaces becomes extremely precise and powerful when carried out with synchrotron radiation, since it provides us the photon energy and light polarization tunability to readily identify molecular levels.

Confirmation of the nature of the probed molecular orbitals is obtained by comparing valence band spectra measured at different excitation energies. The analysis relies on the change of photoionization cross section, ${ }^{25}$ whose decrease with increasing photon energy is much larger for the valence band features arising from $C 2 p$ and $N 2 p$ atomic orbitals than for those arising from the $3 \mathrm{~d}$ atomic orbitals of the Pc metal center, thus changing their relative intensities. ${ }^{26,27}$ The inset of Fig. 2a depicts $\mathrm{F}_{16} \mathrm{CuPc}$ on $\mathrm{Ag}(111)$ valence band spectra measured with photon energies of $21 \mathrm{eV}$ and $140 \mathrm{eV}$, after subtraction of the corresponding substrate reference. The various features are separately labeled and the spectra are normalized to a common intensity of peak A, which corresponds to the macrocycle-based HOMO ( $a_{1 u}$ symmetry) composed mainly by C $2 p_{z}$ orbitals. Peak A' remains almost unchanged, indicating that both states derive from orbitals with a similar atomic character, i.e. $\mathrm{C}$ or $\mathrm{N}$ derived orbitals, in line with our suggested scenario of $\mathrm{A}^{\prime}$ corresponding to a partially filled $\mathrm{e}_{\mathrm{g}}$ LUMO orbital, and not to the partial filling of the $\mathrm{Cu} 3 \mathrm{~d}$ derived singly unoccupied molecular orbital (SUMO). ${ }^{17}$ On the other hand, the increasing intensity in the $140 \mathrm{eV}$ spectrum of feature $\mathrm{C}$ and partially of feature $\mathrm{B}$ [which corresponds to a 
sum of the vibronic loss of the HOMO (peak A) and the split-off band of state C, as observed for $\mathrm{CuPc}$ on $\mathrm{Ag}(111)^{28}$ ] suggests these signals include contributions from $\mathrm{Cu}$-derived orbitals. We therefore ascribe $\mathrm{C}$ to the singly occupied molecular orbital (SOMO, $\mathrm{b}_{1 \mathrm{~g}}$ symmetry), composed mainly by a Cu $3 d_{x^{2}-y^{2}}$ orbital mixed with contributions from the in-plane $2 p_{x, y}$ orbitals of the four surrounding $\mathrm{N}$ atoms. ${ }^{17}$

Regarding the empty molecular states, we obtain important information from the strong linear dichroism exhibited by the NEXAFS spectra (Fig. 2b).${ }^{29}$ Low energy resonances are dominated by transitions into $\pi^{*}$ orbitals, whose intensity is maximized under p-polarization and vanishes under s-polarization. Only one small peak is detected in s-polarization at low photon energies. This spectral feature corresponds to transitions from $\mathrm{N}$ 1s core levels on the inner nitrogen atoms

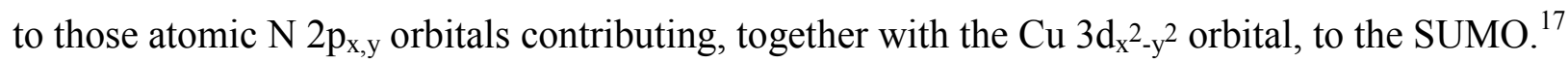
Its slightly higher energy with respect to the main p-polarization resonance completes, together with the previous photoemission results, our deduction of the order by energy of the molecular orbitals. As shown in the inset of Fig. 2b, the SOMO and SUMO are both outside the HOMOLUMO gap. The same molecular orbital order is concluded for CuPc from our combined photon energy dependent photoemission spectra (inset in Fig. 5a) and NEXAFS experiments (Fig. 5b). While this goes against several theoretical predictions, ${ }^{17,26,21}$ other calculations including stronger electron correlations corroborate our findings both in the cases of $\mathrm{CuPc},{ }^{27,30}$ and $\mathrm{F}_{16} \mathrm{CuPc}^{31}$ But most important for the present work is the confirmation that the charge transfer from substrate to the molecules does not flow into the SUMO, but into the degenerate LUMO levels causing their Jahn-Teller splitting. 
The relation of the double core-level components with the observations in NEXAFS spectra needs to be clarified. Split core-levels can arise from initial or final state effects. The former might relate to, for example, the presence of different adsorption sites. The increasing number of components in $\mathrm{C} \mathrm{K}$-edge and $\mathrm{N}$ K-edge NEXAFS spectra could then be interpreted as a result of the double number of initial states for absorption transitions, without invoking the splitting of LUMO levels. However, this should affect transitions into all unoccupied states alike, while unambiguous splitting is observed only in those transitions involving the phthalocyanine's LUMO. Besides, according to this assumption, the equal intensity of the split components in XPS spectra would indicate the presence of two equally populated and well defined initial states, which in turn imply ordered molecular arrangements. Focusing for example on the case of $\mathrm{CuPc} / \mathrm{Ag}(111)$, the core-level splitting observed in our data (Fig. 5c) would point to an ordered structure already at the submonolayer coverage. However, this interpretation goes against reports of Kroger et al., who observe that CuPc molecules below 0.89 ML display no crystalline order and behave like a $2 \mathrm{D}$ gas at room temperature, ${ }^{28}$ discarding again the hypothesis that the splitting of core-levels is an initial-state effect and causes the doubling of NEXAFS transitions.

We suggest an alternative interpretation that does not contradict the observations above: a lifted degeneracy of the LUMO level upon partial charging in combination with core-hole screening effects as the origin of the doubled core-levels. The latter are related to the different screening associated with the delocalized charge throughout the LUMO. As displayed in Fig. 1c, the degenerate LUMO levels extend along different molecular diagonals and partial charging of one of them affects half of each of the atomic species in the molecule [this effect is not observed on the F atoms, which as shown in Fig. 1c hardly participate of the LUMO level and therefore leave its core levels virtually unaffected (Fig. S3)]. While the split core-level components at 
higher binding energy nearly follow the work function shift of the system, their counterparts benefit from the more effective screening of the partially filled LUMO and are found at lower binding energy. The efficiency of electron screening depends on the degree of LUMO filling, i.e. major screening effects are observed for the system with the largest amount of transferred charge. This is again the case of $\mathrm{F}_{16} \mathrm{CuPc}: \mathrm{PEN} / \mathrm{Cu}(111)$, where the energy separation of the corelevel doublets is largest in both $\mathrm{C} 1 \mathrm{~s}$ and $\mathrm{N}$ 1s spectra. Because NEXAFS transitions don't ionize the molecules, screening or final state effects hardly affect the absorption spectra and the splitting can be directly ascribed to the lifted degeneracy of the LUMO levels.

Proof and direct observation of the Jahn-Teller effect upon phthalocyanine charging has been obtained by scanning tunneling microscopy and spectroscopy on CuPc charged externally by the scanning probe. ${ }^{16}$ The LUMO splitting was claimed to amount to $0.21 \mathrm{eV}$, and although this value is significantly smaller than the $\sim 0.7 \mathrm{eV}$ estimated from the analysis of Fig. $5(\sim 0.55 \mathrm{eV}$ between main and side maxima in NEXAFS spectra, plus $0.15 \mathrm{eV}$ binding energy of the FLUMO intensity maximum in the valence band spectrum), this discrepancy might arise from the different characterization techniques, as well as from the effect of considerably different substrates [an insulating $\mathrm{NaCl}$ bilayer on $\mathrm{Cu}(111)$ in ref. 16 vs. $\mathrm{Ag}(111)$ in the present work] . Overall, the observed Jahn-Teller effect in singly charged CuPc molecules provides strong support for our interpretation of the spectroscopic fingerprints upon partial charge transfer to $\mathrm{CuPc}$ and $\mathrm{F}_{16} \mathrm{CuPc}$, which we have further shown to be tunable even for one given intimate molecule-substrate interface.

\section{Methods}


The $\mathrm{Au}(111), \operatorname{Ag}(111)$, and $\mathrm{Cu}(111)$ surfaces were prepared by standard $\mathrm{Ar}$ sputtering and annealing cycles, and their cleanliness checked by XPS or STM prior to molecular deposition. $\mathrm{CuPc}, \mathrm{F}_{16} \mathrm{CuPc}$, and PEN were purchased from Sigma-Aldrich. The molecules have been used as received, except $\mathrm{F}_{16} \mathrm{CuPc}$, which was additionally purified by gradient sublimation. The molecular layers were prepared by the deposition from resistively heated Knudsen cells at temperatures around $380^{\circ} \mathrm{C}$ and $190{ }^{\circ} \mathrm{C}$ for the phthalocyanines and pentacene, respectively, onto single crystal surfaces held at room temperature. The sample coverage was monitored by means of a quartz crystal microbalance and further corroborated by detailed analysis of the relative core-level peak intensities. Potential beam damage on the films was minimized when necessary by moving the photon beam across the sample. Beam damage as the source of spectral changes was discarded by checking the reproducibility of spectra after irradiation.

All measurements were performed at room temperature under ultrahigh vacuum (UHV) conditions. NEXAFS and XPS experiments were performed at the ALOISA beamline of the synchrotron light source ELETTRA in Trieste (Italy). NEXAFS spectra were taken in partial electron yield by means of a channeltron detector. Spectra measured at the C and N K-edges were calibrated by acquisition of the $1 \mathrm{~s}-\pi^{*}$ gas phase transitions of $\mathrm{CO}$ and $\mathrm{N}_{2}$ at $\mathrm{h} v=287.4 \mathrm{eV}$ and $401.10 \mathrm{eV}$, respectively. ${ }^{32}$ The manipulator is coaxial to the photon beam, which allows to change the orientation of the surface with respect to linear polarization of the beam while keeping constant the grazing angle, i.e., without changing the beam footprint on the sample. For more details about the scattering geometry, see Ref. 33 . In order to compare the intensity of the spectra taken on the different samples, the spectra have been normalized to the intensity at high photon energy, i.e. $\mathrm{h} v=305 \mathrm{eV}$ for the $\mathrm{C} \mathrm{K}$-edge and $\mathrm{h} v=420 \mathrm{eV}$ for the $\mathrm{N}$ K-edge. 
The XPS data were collected by means of a hemispherical electron energy analyzer in normal emission while keeping the sample at grazing incidence $\left(\sim 4^{\circ}\right)$, with excitation energies of $140 \mathrm{eV}$ (valence band) and $530 \mathrm{eV}(\mathrm{C} 1 \mathrm{~s}, \mathrm{~N} 1 \mathrm{~s})$ and overall resolution of $120 \mathrm{meV}$ and $240 \mathrm{meV}$, respectively. The binding energy of core-level spectra is carefully calibrated taking the substrate core-level energies as absolute references. The fitting of all XPS spectra was done using a Shirley background and Voigt integral functions. In all C 1s and N 1s spectra, the Lorentzian width, FWHM, BEs intensity ratio among the different components are left as free parameters of the fit. In $\mathrm{C} 1 \mathrm{~s}$ and $\mathrm{N} 1 \mathrm{~s}$ spectra of $\mathrm{F}_{16} \mathrm{CuPc} / \mathrm{Ag}(111), \mathrm{F}_{16} \mathrm{CuPc}: \mathrm{PEN} / \mathrm{Cu}(111)$ the intensity ratio of the double components results close to unity within $15 \%$.

Ultraviolet photoemission spectroscopy measurements were recorded $45^{\circ}$ off-normal emission.Valence band spectra of $\mathrm{F}_{16} \mathrm{CuPc} / \mathrm{Au}(111)$ and $\mathrm{F}_{16} \mathrm{CuPc} / \mathrm{Ag}(111)$ were measured using the He I line $(21.2 \mathrm{eV})$ from a non-monochromatized gas discharge lamp and a SPECS Phoibos 100 electron analyzer. The He I satellites lines ( $\beta$ and $\gamma$ ) caused by the non-monochromatized photon source were also taken into account by reducing the intensity of the spectra by $2 \%$ and $0.4 \%$ at fixed kinetic energy intervals of $1.87 \mathrm{eV}$ and $2.52 \mathrm{eV}$, respectively, from the emission associated with the $\mathrm{He} \mathrm{I} \alpha$ main line. This data treatment removes the substrate $d$-band satellites in the vicinity of the photoemission peaks of $\mathrm{F}_{16} \mathrm{CuPc}$. The fit procedure of the valence band spectra was then made using a Shirley background and Voigt integral functions. Valence band spectra of monolayers and blends on $\mathrm{Cu}(111)$ were acquired using the $\mathrm{He} \mathrm{I} \alpha$ line from a monochromatized gas discharge lamp and a SPECS Phoibos 150 electron analyzer with energy and angle resolutions of $\approx 40 \mathrm{meV}$ and $0.1^{\circ}$, respectively.

The work function was determined by the low energy cut-off in the photoemission spectra (sample bias $24 \mathrm{~V}$ ). STM images were measured at room temperature in a commercial JEOL 
STM system in constant current mode. The analysis of the STM images has been performed with the freeware WSxM from Nanotec. ${ }^{34}$ Calculation details regarding the displayed molecular orbitals of $\mathrm{F}_{16} \mathrm{CuPc}$ are provided in reference 21 .

\section{Acknowledgements}

This work was supported by the Spanish Grants No. MAT2010-21156-C03-01, PIB2010US00652, and the Basque Government Grant No. IT-621-13. We acknowledge funding from the European Community's Seventh Framework Programme (FP7/2007-2013) under Grant No. 226716. We thank J.-M. Garcia-Lastra for interesting discussions, G. Lovat, A. Verdini and A. Cossaro for their support during beamtimes, Y. Wakayama for his support with STM measurements, and D. Pickup for his help with home-laboratory photoemission measurements.

\section{Author contributions}

"P.B., C.R., J.E.O and D.G.O. conceived and designed the experiments; P. B., A.E.-S., E.G. C.R., J.L.-C., L.F., and D.G.O. performed the experiments; P.B., A.E.-S., and E.G. analyzed the data; P.B., J.E.O. and D.G.O. co-wrote the paper and all co-authors assisted in its editing." Additional information

The authors declare no competing financial interests. Supplementary information accompanies this paper at www.nature.com/naturechemistry. Reprints and permission information is available online at http://npg.nature.com/reprintsandpermissions/. Correspondence and requests for materials should be addressed to P.B., J.E.O and D.G.O. 


\section{Figures:}
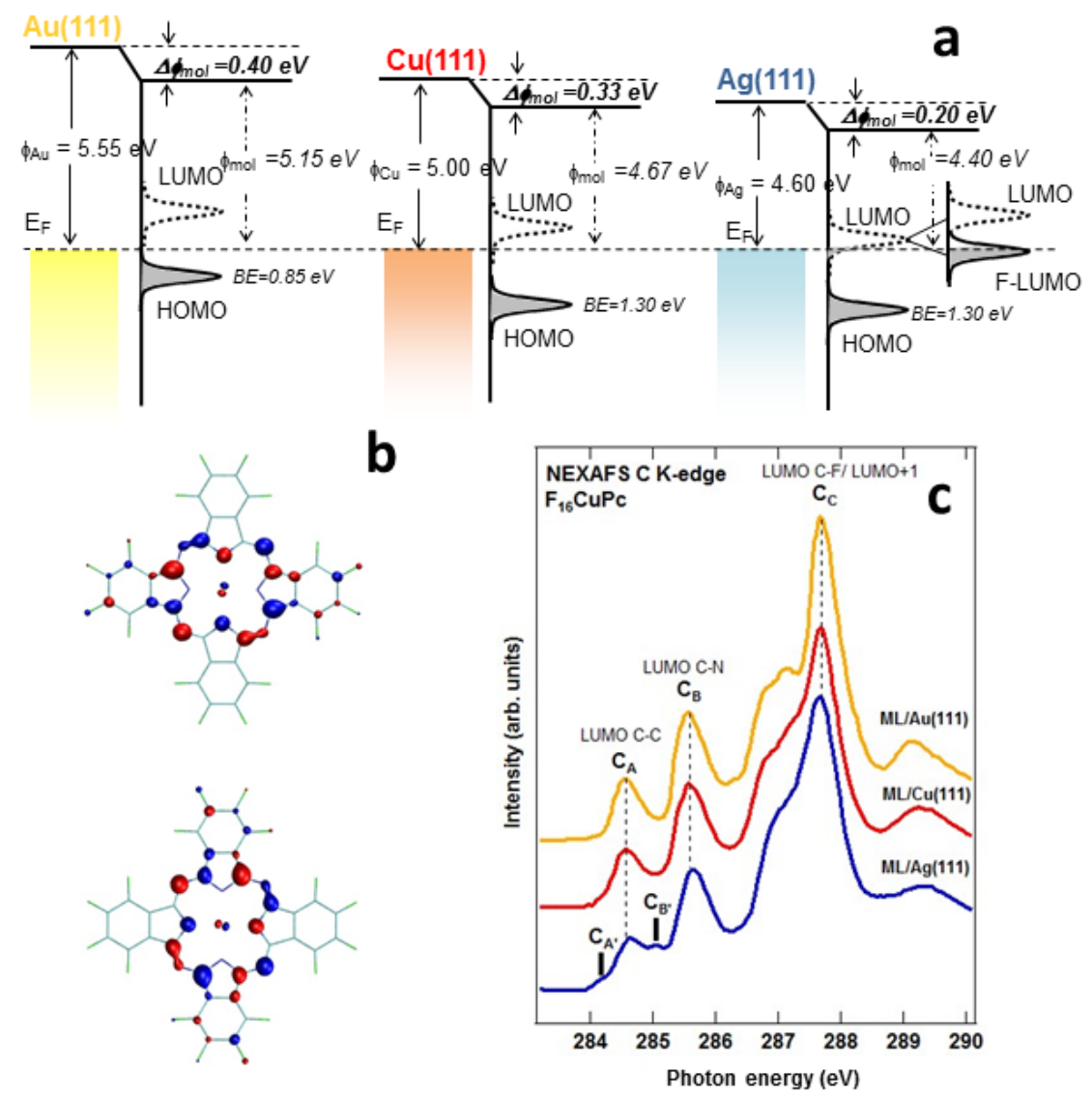

Fig. 1. Interface energetics of $\mathrm{F}_{16} \mathrm{CuPc}$ on coinage metal surfaces of varying work-function, reaching a partial charge transfer scenario with splitting of degenerate LUMO levels.

a. Energy level diagram of a $\mathrm{F}_{16} \mathrm{CuPc}$ monolayer on $\mathrm{Au}(111), \mathrm{Cu}(111), \mathrm{Ag}(111)$ based on the present results. In $\mathrm{F}_{16} \mathrm{CuPc} / \mathrm{Ag}(111), E_{F}$ crosses the low energy tail of the LUMO level, leading to a partial charge transfer from substrate to molecule. As a consequence, the two-fold degeneracy of $\mathrm{F}_{16} \mathrm{CuPc} e_{g} \mathrm{LUMO}(\mathrm{c})$ is lifted and one of the split states is partially occupied upon charging. b. Density of States of the double degenerate $e_{g}$ LUMO levels of the isolated $\mathrm{F}_{16} \mathrm{CuPc}$ molecule (extracted from the calculations of ref. 21). The two orbitals are equal but rotated by $90^{\circ}$ with respect to each other. c. P-polarization NEXAFS C K-edge spectra of $\mathrm{F}_{16} \mathrm{CuPc}$ monolayers on the three surfaces. Transitions to $\mathrm{F}_{16} \mathrm{CuPc}$ LUMO from the $\mathrm{C} 1 \mathrm{~s}$ corelevel at the C-C, C-N, C-F sites are indicated on top of the corresponding peaks. For $\mathrm{F}_{16} \mathrm{CuPc}$ $\operatorname{IAg}(111)$, the presence of additional components due to the LUMO splitting are indicated by green arrows. 


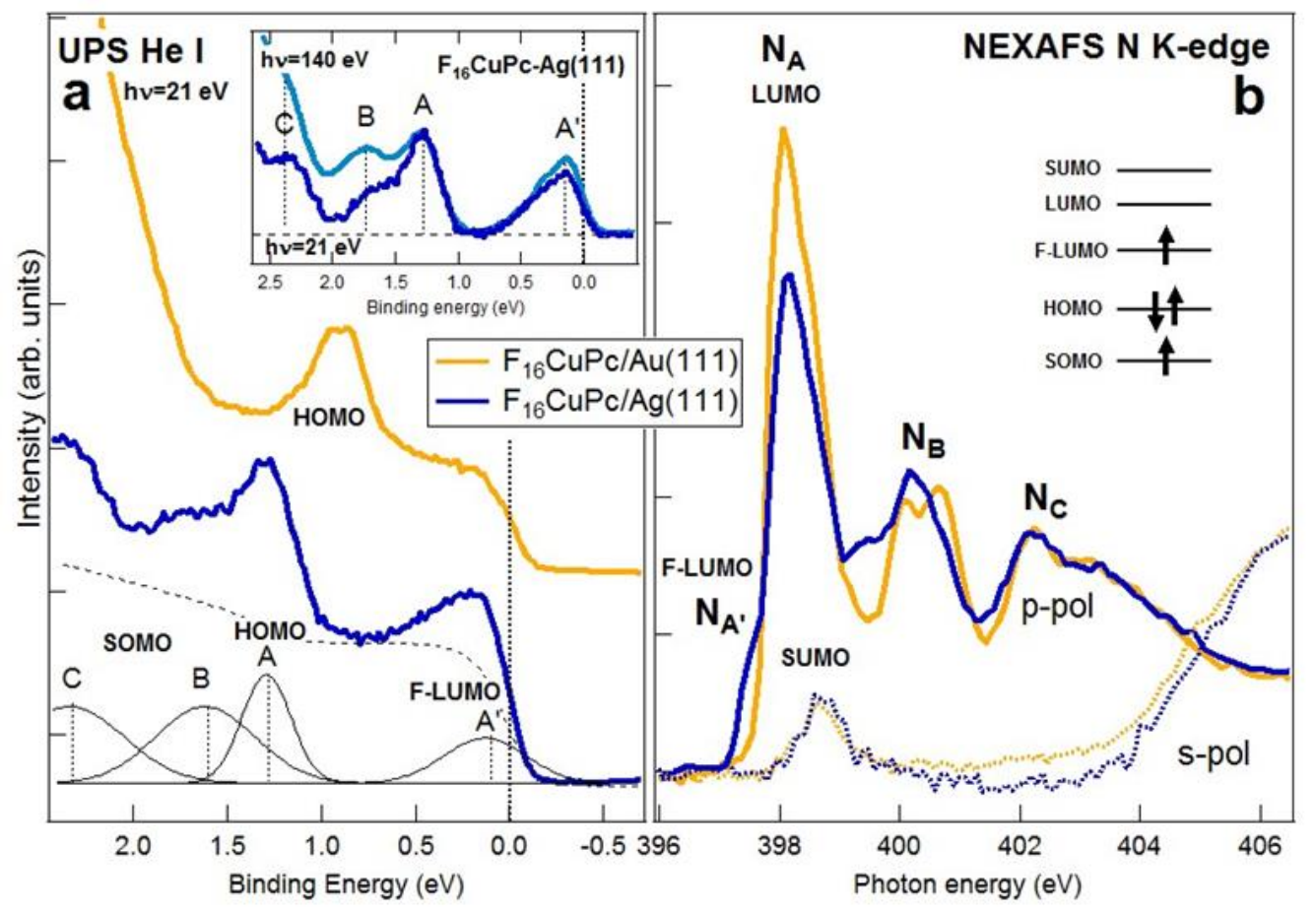

Fig. 2. Analysis of occupied and unoccupied molecular orbitals providing spectroscopic evidence of the partial charge transfer and LUMO level splitting.

a. Valence band spectra of $\mathrm{F}_{16} \mathrm{CuPc} / \mathrm{Au}(111)$ (yellow line) and $\mathrm{F}_{16} \mathrm{CuPc} / \mathrm{Ag}(111)$ (dark blue line) measured with $h v=21 \mathrm{eV}$. For the latter, the fit components (black solid lines) and the Shirley background convoluted with a Fermi step function (black dashed line) obtained from the fitting procedure are shown in the bottom part. Inset: Valence spectra of $\mathrm{F}_{16} \mathrm{CuPc} / \mathrm{Ag}(111)$ taken with $\mathrm{h} v=21 \mathrm{eV}$ (dark blue line) and $\mathrm{h} v=140 \mathrm{eV}$ (light blue line) after subtracting the correspondent clean reference spectra. b. NEXAFS N K-edge spectra of $\mathrm{F}_{16} \mathrm{CuPc} / \mathrm{Au}(111)$ (yellow line) and $\mathrm{F}_{16} \mathrm{CuPc} / \mathrm{Ag}(111)$ (dark blue line) taken both under p-polarization (solid lines) and s-polarization (dashed lines). Inset: Indicative energy scheme of $\mathrm{F}_{16} \mathrm{CuPc}$ energy levels upon charging, as deduced from present data. 


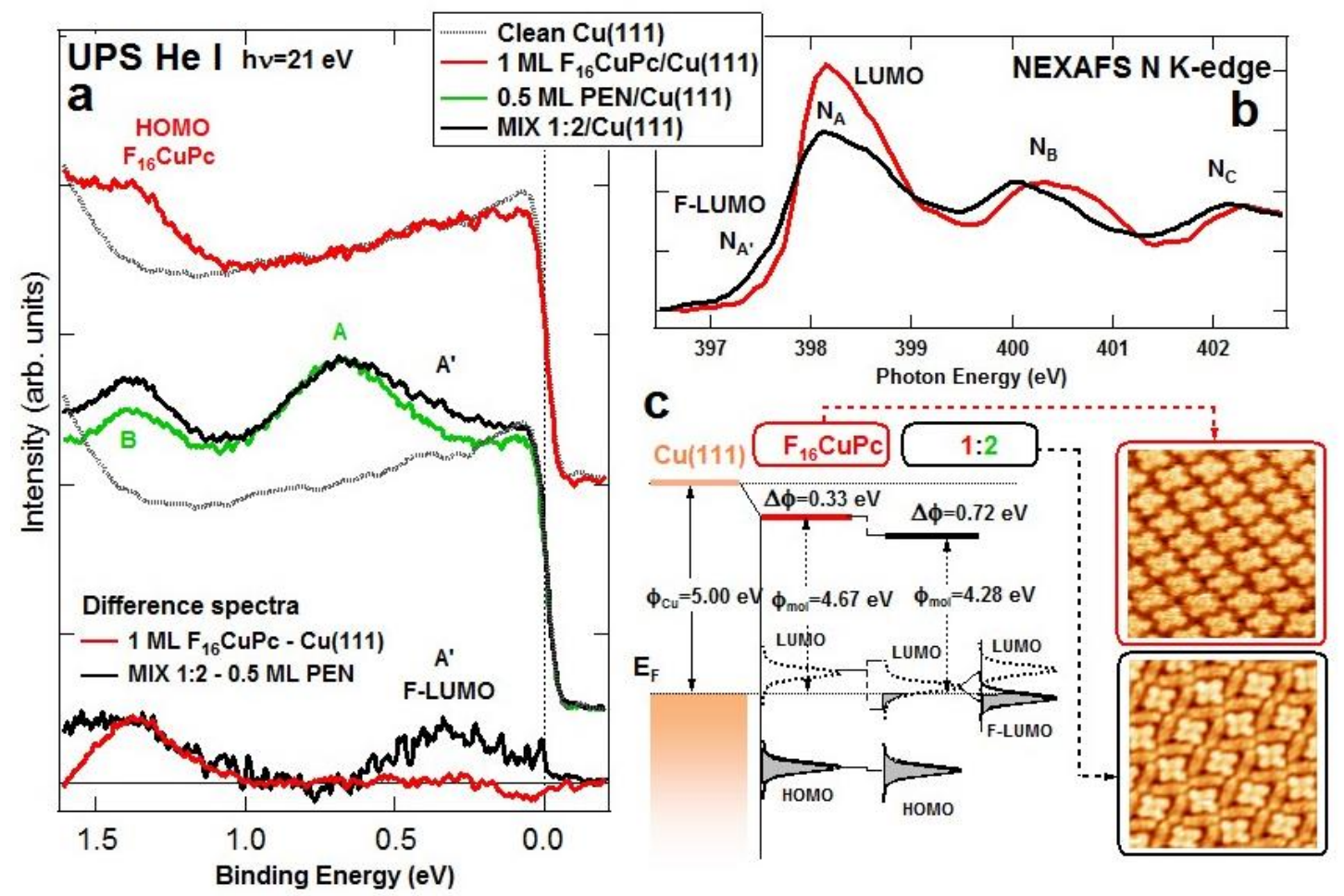

Fig. 3. Controllable charging of $\mathrm{F}_{16} \mathrm{CuPc}$ on $\mathrm{Cu}(111)$ by work-function manipulation through its supramolecular environment.

a. Valence band spectra of a monolayer of $\mathrm{F}_{16} \mathrm{CuPc} / \mathrm{Cu}(111)$ (top red line), $0.5 \mathrm{ML}$ of $\mathrm{PEN} / \mathrm{Cu}(111)$ (middle green line) and the subsequent 1:2 blend of $\mathrm{F}_{16} \mathrm{CuPc}: \mathrm{PEN} / \mathrm{Cu}(111)$ (middle black line). $\mathrm{Cu}(111)$ clean reference spectra are also shown (grey dotted lines). The bottom part displays the $\mathrm{F}_{16} \mathrm{CuPc} / \mathrm{Cu}(111)$ spectrum after subtracting the clean reference spectrum (red line) and the 1:2 blend spectrum after subtracting the 0.5 ML PEN spectrum (black line). In order to account for the reduced amount of $\mathrm{F}_{16} \mathrm{CuPc}$, the spectrum of the mix has been doubled in intensity, which consistently results in an equal intensity value for the $\mathrm{F}_{16} \mathrm{CuPc}$ HOMO peak. b. NEXAFS N K-edge spectra of $\mathrm{F}_{16} \mathrm{CuPc} / \mathrm{Cu}(111)$ (red line) and the 1:2 blend of $\mathrm{F}_{16} \mathrm{CuPc}: \mathrm{PEN} / \mathrm{Cu}(111)$ (black line) acquired in p-polarization. For both systems, the schematic energy level diagram and STM images $\left(9 \times 9 \mathrm{~nm}^{2}, \mathrm{~V}=-0.7 \mathrm{~V}\right.$ and $\left.\mathrm{I}=0.7 \mathrm{nA}\right)$ in constant current mode are presented in panel c. As sketched, the work function reduction in the 1:2 blend leads to the partial charge transfer from substrate to $\mathrm{F}_{16} \mathrm{CuPc}$ molecule, and hence to the splitting of the two-fold degenerate $\mathrm{F}_{16} \mathrm{CuPc}$ LUMO levels. 


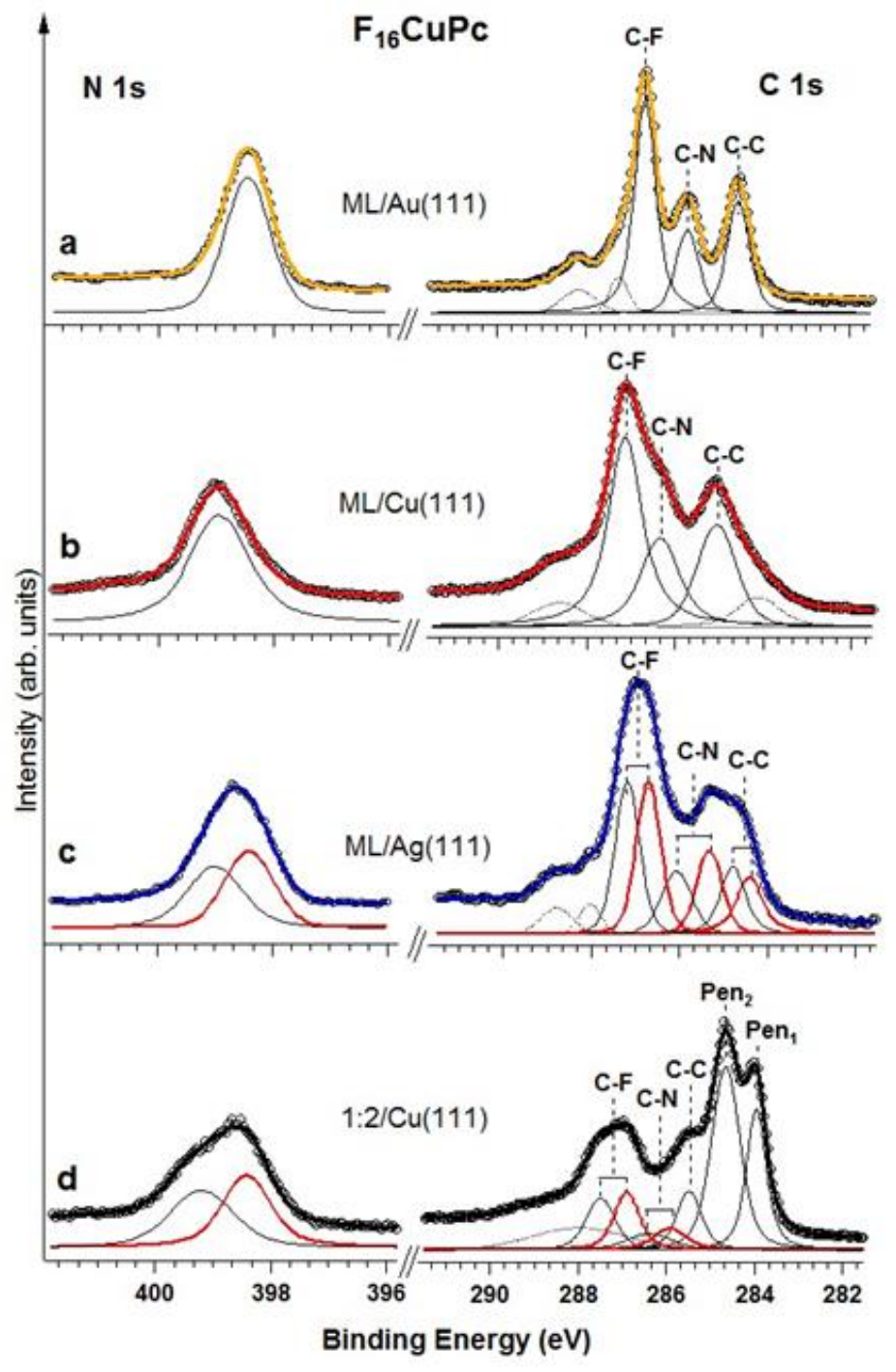

Fig. 4. $\mathrm{F}_{16} \mathrm{CuPc}$ core level fotoemission spectra on varying work-function substrates.

X-ray photoemission spectra in the $\mathrm{N}$ 1s (left panels) and C 1s (right panels) core-level regions of $\mathrm{F}_{16} \mathrm{CuPc} / \mathrm{Au}(111)(\mathbf{a}), \mathrm{F}_{16} \mathrm{CuPc} / \mathrm{Cu}(111)(\mathbf{b}), \mathrm{F}_{16} \mathrm{CuPc} / \mathrm{Ag}(111)(\mathbf{c})$ and 1:2 blend of $\mathrm{F}_{16} \mathrm{CuPc}: \mathrm{PEN} / \mathrm{Cu}(111)(\mathbf{d})$. Experimental points (markers) are superimposed to fit spectra (solid line). In (c) and (d) split-off components (colored in red) arise as a consequence of the more efficient screening of the partially filled LUMO. 


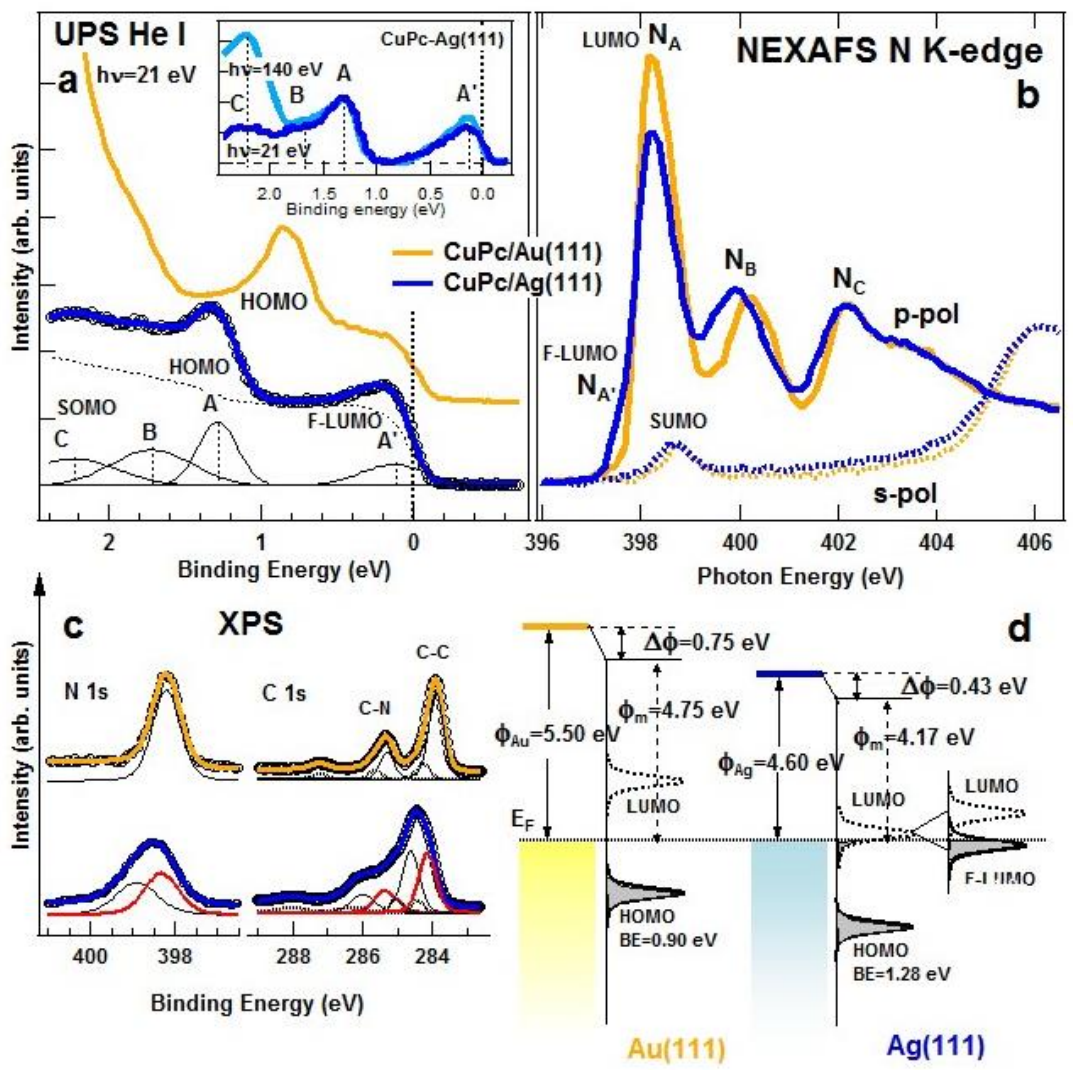

Fig. 5. Comparing the phenomenology for the closely related molecule CuPc.

a. Valence band spectra of $\mathrm{CuPc} / \mathrm{Au}(111)$ (yellow line) and $\mathrm{CuPc} / \mathrm{Ag}(111)$ (dark blue line) measured with $h v=21 \mathrm{eV}$. For the latter, the fit components (black solid lines) and the Shirley background convoluted with a Fermi step function (black dashed line) obtained from the fitting procedure are shown below. Inset: Valence spectra of CuPc/Ag(111) taken with hv=21 eV (dark blue line) and $h v=140 \mathrm{eV}$ (light blue line) after subtracting the correspondent clean reference spectra. b. NEXAFS N K-edge spectra of of CuPc/Au(111) (yellow line) and CuPc/Ag(111) (dark blue line) acquired both under p-polarization (solid lines) and s-polarization (dashed lines). c. X-ray photoemission spectra in the $\mathrm{N} 1 \mathrm{~s}$ (left panels) and $\mathrm{C} 1 \mathrm{~s}$ (right panels) core-level regions of $\mathrm{CuPc} / \mathrm{Au}(111)$ (top spectra) and $\mathrm{CuPc} / \mathrm{Ag}(111)$ (bottom spectra). Experimental points (markers) are superimposed to fit spectra (solid line). As in Fig. 4, the split-off components arising from the partial LUMO filling are colored in red. d. Energy level diagrams for $\mathrm{CuPc} / \mathrm{Au}(111)$ and $\mathrm{CuPc} / \mathrm{Ag}(111)$. For the latter, $\mathrm{E}_{\mathrm{F}}$ crosses the low energy LUMO tail, inducing a partial charge transfer from substrate to $\mathrm{CuPc}$ and the splitting of the two-fold degenerate $\mathrm{CuPc}$ LUMO levels. 


\section{References}

${ }^{1}$ Marmont P., Battaglini N., Lang P., Horowitz G., Hwang J., Kahn A., Amato C., Calas P., Improving charge injection in organic thin-film transistors with thiol-based self-assembled monolayers. Org. Electron. 9, 419-424 (2008)

${ }^{2}$ Ziroff., J., Foster, F., Schöll, A., Puschnig, P., Reinert, F. Hybridization of Organic Molecular Orbitals with Substrate States at Interfaces: PTCDA on Silver. Phys. Rev. Lett. 104, 233004 (2010)

${ }^{3}$ Heimel, G., et al. Charged and metallic molecular monolayers through surface-induced aromatic stabilization. Nat. Chem. 5, 187-194 (2013)

${ }^{4}$ Salzmann, I., Heimel, G., Duhm, S., Oehzelt, M., Pingel, P., George, B.M., Schneeg, A., Lips, K., Blum, R.-P., Vollmer, A., Koch, N. Intermolecular Hybridization Governs Molecular Electrical Doping. Phys. Rev. Lett. 108, 035502-1-5 (2012)

${ }^{5}$ Koch, N., Duhm, S., Rabe, J.P., Vollmer, A., Johnson, R.L. Optimized Hole Injection with Strong Electron Acceptors at Organic-Metal Interfaces. Phys. Rev. Lett. 95, 237601-1-4 (2005)

${ }^{6}$ Tseng, C.-T., Cheng, Y.-H., Lee, M.-C. M., Han, C.-C., Cheng C.-H., Tao, Y.-T. Study of anode work function modified by self-assembled monolayers on pentacene/fullerene organic solar cells. Appl. Phys. Lett. 91 , 233510-1-3 (2007)

${ }^{7}$ Hong, J.-P., Park, A.-Y., Lee, S., Kang, J., Shin N., Yoon, D. Y. Tuning of Ag work functions by self-assembled monolayers of aromatic thiols for an efficient hole injection for solution processed triisopropylsilylethynyl pentacene organic thin film transistors. Appl. Phys. Lett. 92 , $143311-1-3(2008)$ 
${ }^{8}$ Jia, Z., Lee, V.W., Kymissis, I., Floreano, L., Verdini, A., Cossaro, A., Morgante, A. In situ study of pentacene interaction with archetypal hybrid contacts: Fluorinated versus alkane thiols on gold. Phys. Rev. B 82, 125457-1-6 (2010)

${ }^{9}$ Monch, W. On the physics of metal-semiconductor interfaces. Rep. Prog. Phys. 53, 221-278 (1990)

${ }^{10}$ Tung, R. T. The physics and chemistry of the Schottky barrier height. Appl. Phys. Rev. 1, 011304-1-54 (2014)

${ }^{11}$ Hwang, J., Wan, A., Kahn, A. Energetics of metal-organic interfaces: New experiments and assessment of the field. Mat. Sci. Eng. R 64, 1-31 (2009)

${ }^{12}$ Flores, F., Ortega, J., Vazquez, H. Modelling energy level alignment at organic interfaces and density functional theory. Phys. Chem. Chem. Phys. 11, 8658-8675 (2009)

${ }^{13}$ Peisert, H., Knupfer, M., Schwieger, T., Fuentes, G.G., Olligs, D., Fink, J., Schmidt, Th. Fluorination of copper phthalocyanines: Electronic structure and interface properties. J. Appl. Phys. 93, 9683-9692 (2003)

${ }^{14}$ El-Sayed, A., et al. Understanding energy level alignment in donor-acceptor/metal interfaces from core-level shifts. ACS Nano 7, 6914-6920 (2013)

${ }^{15}$ Goiri, E., Matena, M., El-Sayed, A., Lobo-Checa, J., Borghetti, P., Detlefs, B., Duvernay, J., Ortega, J.E., de Oteyza, D.G. Self-Assembly of Bicomponent Molecular Monolayers: Adsorption Height Changes and Their Consequences. Phys. Rev. Lett. 112, 117602-1-5 (2014)

${ }^{16}$ Uhlmann, C., Swart, I., Repp, J. Controlling the Orbital Sequence in Individual CuPhthalocyanine Molecules, Nano Lett. 13, 777-780 (2013) 
${ }^{17}$ Piper, L. F. J., Cho, S. W., Zhang, Y., DeMasi, A., Smith, K. E., Matsuura, A. Y., Mc.

Guinness, C. Soft x-ray spectroscopy study of the element and orbital contributions to the electronic structure of copper hexadecafluoro-phthalocyanine, Phys. Rev. B 81, 045201 (2010)

${ }^{18}$ Calabrese, A., Floreano, L., Verdini, A., Mariani, C., Betti, M.G. Filling empty states in a CuPc single layer on the Au(110) surface via electron injection. Phys. Rev. B. 79, 115446-1-7 (2009)

${ }^{19}$ Evangelista, F., Gotter, R., Mahne, N., Nannarone, S., Ruocco, A., Rudolf, P. Electronic Properties and Orbital-Filling Mechanism in Rb-Intercalated Copper Phthalocyanine. J. Phys. Chem. C 112, 6509-6514 (2008)

${ }^{20}$ Wakayama, Y., de Oteyza, D. G., Garcia-Lastra, J. M., Mowbray, D. J. Solid-state reactions in binary molecular assemblies of $\mathrm{F}_{16} \mathrm{CuPc}$ and Pentacene. ACS Nano 5, 581-589 (2011)

${ }^{21}$ de Oteyza, D. G., et al. Copper-phthalocyanine based metal-organic interfaces: The effect of fluorination, the substrate, and its symmetry. J. Chem. Phys. 133, 214703-1-6 (2010)

${ }^{22}$ Cabellos, J.L., Mowbray, D.J., Goiri, E., El-Sayed, A., Floreano, L., de Oteyza, D.G., Rogero, C., Ortega, J.E., Rubio, A. Understanding Charge Transfer in Donor-Acceptor/Metal Systems: A Combined Theoretical and Experimental Study. J. Phys. Chem. C 116, 17991-18001 (2012)

${ }^{23}$ Koch, N., et al. Adsorption-Induced Intramolecular Dipole: Correlating Molecular Conformation and Interface Electronic Structure. J. Am. Chem. Soc. 130, 7300-7304 (2008)

${ }^{24}$ Babudri, F., Farinola, G. M., Naso, F., Ragni, R. Fluorinated organic materials for electronic and optoelectronic applications: the role of the fluorine atom. Chem. Commun. 1003-1022 (2007)

${ }^{25}$ Yeh, J. J., Lindau, I. Atomic subshell photoionization cross sections and asymmetry parameters. At. Data Nucl. Data Tables 32, 1-155 (1985) 
${ }^{26}$ Evangelista, F., Carravetta, V., Stefani, G., Jansik, B., Alagia, M., Stranges, S., Ruocco, A. Electronic structure of copper phthalocyanine: An experimental and theoretical study of occupied and unoccupied levels. J. Chem. Phys. 126, 124709-1-10 (2007)

${ }^{27}$ Nardi, M.V., Detto, F., Aversa, L., Verucchi, R., Salviati, G., Iannotta, S., Casarin, M. Electronic properties of $\mathrm{CuPc}$ and $\mathrm{H}_{2} \mathrm{Pc}$ : an experimental and theoretical study. Phys. Chem. Chem. Phys. 15, 12864-12881 (2013)

${ }^{28}$ Kroger, I., et al. Submonolayer growth of copper-phthalocyanine on $\mathrm{Ag}(111)$. New J. Phys. 12, 083038-1-23 (2010)

${ }^{29}$ de Oteyza, D.G., Sakko, A., El-Sayed, A., Goiri, E., Floreano, L., Cossaro, A., Garcia-Lastra, J.M., Rubio, A., Ortega, J. E., Inversed linear dichroism in F K-edge NEXAFS spectra of fluorinated planar aromatic molecules. Phys. Rev. B 86, 075469-1-6 (2012)

${ }^{30}$ Aristov, V.Y., et al. Electronic structure of pristine CuPc: Experiment and calculations. Appl. Surf. Sci. 254, 20-25 (2007)

${ }^{31}$ Ren, J., Meng, S., Wang, Y.-L., Ma, X.-C., Xue, Q.-K., Kaxiras, E. Properties of copper (fluoro-)phthalocyanine layers deposited on epitaxial graphene. J. Chem. Phys. 134, 194706 (2011)

${ }^{32}$ Floreano, L., Naletto, G., Gvetko, D., Gotter, R., Malvezzi, M., Marassi, L., Morgante, A., Santaniello, A., Verdini, A., Tommasini, F., Tondello, G. Performance of the grating-crystal monochromator of the ALOISA beamline at the Elettra Synchrotron. Rev. Sci. Instrum. 70, 38553864 (1999)

${ }^{33}$ Floreano, L., Cossaro, A., Gotter, R., Verdini, A., Bavdek, G., Evangelista, F., Ruocco, A., Morgante, A., Cvetko, D. Periodic Arrays of Cu-Phthalocyanine Chains on Au(110). J. Phys. Chem. C 112, 10794-10802 (2008) 
${ }^{34}$ Horcas, I., Fernandez, R., Gomez-Rodriguez, J.M., Colchero, J., Gomez-Herrero, J., Baro, A.M. WSXM: A software for scanning probe microscopy and a tool for nanotechnology. Rev. Sci. Instrum. 78, 013705-1-8 (2007) 